[0212-7199 (2004) 21: 11; pp 540-542] ANALES DE MEDICINA INTERNA Copyright (C) 2004 ARAN EDICIONES, S.L.

AN. MED. InTERNA (Madrid) Vol. 21, N. $^{\circ} 11$, pp. $540-542,2004$

\section{Especialidades farmacéuticas genéricas en un hospital comarcal. Impacto de dos estrategias de intervención}

\author{
C. TORNERO ESTÉBANEZ, E. QUINTANA GALLEGO'1, J. A. LULL SALA, \\ I. PÉREZ CASTELLO ${ }^{1}$, M. SINTES MARCO, E. SOLER COMPANY ${ }^{1}$
}

Servicio de Medicina Interna $y^{\prime}$ Farmacia. Hospital Francisco de Borja. Gandía. Valencia
GENERIC DRUGS IN A DISTRIC HOSPITAL. INTERVENTION STRATEGIES

\section{RESUMEN}

Objetivo: Conocer los hábitos de prescripción de especialidades farmacéuticas genéricas en atención hospitalizada y el impacto de dos diferentes estrategias de intervención.

Material y métodos: Se diseña un estudio de intervención con evaluación previa y comparación posterior. A partir de los informes de alta generados en el Hospital Comarcal Francisco de Borja de Gandía se recogieron los fármacos prescritos, la proporción de ellos en los que existe alternativa como EFG disponible y porcentaje que se pauto como tal. Tras una notar informativa de carácter general se repitió la toma de datos al mes. En el Servicio de Medicina Interna se analizó también el perfil presciptor de cada facultativo, redactándose una hoja informativa personalizada que se distribuyo de forma personal, acompañada de una breve explicación verbal. Se repitió la toma de datos a la semana y al mes.

Resultados: Se revisaron un total de 1545 informes de alta y 3460 fármacos prescritos. En la toma de datos basal el $42 \%$ de la medicación prescrita podría haberse pautado como EFG pero se prescribió como tal en el $10 \%$ (21\% en el Servicio de Medicina Interna).

Tras la nota informativa general no se objetivaron variaciones significativas en el porcentaje de utilización de EFG. En Medicina Interna, se obtuvo un significativo incremento a la semana de la intervención, del $21 \%$ se paso al $156,8 \%$ (RR 0.38 IC al $95 \%(0,26-0,55)$ pero que tendía a desvanecerse al mes (27\%) perdiendo significación estadística.

Comentarios: La utilidad de campañas de promoción del uso de EFG es limitada y transitoria por lo que además de mejorar la información respecto a la disponibilidad y eficacia de las EFG, se debería contemplar la implicación del prescriptor tanto en la atención primaria como en la especializada.

PALABRAS CLAVE: Fármacos genéricos. Campañas de promoción. Gasto sanitario.

\section{ABSTRACT}

Objective: To know the prescription of generic drugs in specialized attention and the impact of two different intervention strategies:

Material and methods: Intervention trial with evaluation and later comparison. The data on the use of generic drugs are obtained from the medical reports. After an informative note of general character we repeated the taking of data one month later. In the service of Medicine we also analysed each doctor's profile prescriptions and a personalized informative note was edited and accompanied by a brief verbal explication of the campaign. Then repeated the collection of data to the week and the month.

Results: 1545 reports were revised (3460 prescribed drugs). $42 \%$ of the medication could be prescribed as generic but it was only made in $10 \%$ (21\% in Internal Medicine). After the informative note no significant variations were observed. Only in Internal Medicine an initial increment was obtained $(21 \%$ to $56 \%$, RR 0,38, IC 0.26-0.55) that it disappeared at month.

Comments: The utility of the intervention to generic promotion is limited and transitory. Besides improve the information, semms necessary the implication of the physician in the promotion campaigns

KEY WORDS: Generic drugs. Promotion campaigns. Drugs cost.

Tornero Estébanez, C, Quintana Gallego E, Lull Sala JA, Pérez Castello I, Sintes Marco M, Soler Company E. Especialidades farmacéuticas genéricas en un hospital comarcal. Impacto de dos estrategias de intervención. An Med Interna (Madrid) 2004; 21: 540-542.

\section{INTRODUCCIÓN}

Las especialidades farmacéuticas genéricas (EFG) se han introducido progresivamente en los países de nuestro entorno como parte de políticas dirigidas a la contención del gasto sanitario.
En la promoción de su uso se debería implicar a la atención especializada, dada la repercusión que puede tener sobre la atención primaria y el gasto farmacéutico. Sin embargo no son abundantes las iniciativas orientadas a promover su utilización en las pautas de tratamiento generadas en el entorno hospitalario, pero que se mantienen al alta del paciente.

Trabajo aceptado: 29 de junio de 2004 
Nos proponemos conocer el porcentaje de utilización de EFG en un hospital comarcal, las razones de mayor o menor uso, y el efecto que se obtiene con diferentes estrategias de promoción.

\section{MATERIAL Y MÉTODO}

Se diseña un estudio de intervención con evaluación previa y comparación posterior, con análisis de frecuencias y porcentajes, y comparación mediante test de la CHI cuadrado. Se utilizó el programa EPI info 2000 y el criterio de decisión para la significación estadística se estableció en un nivel de $\mathrm{a}=0.05$ o inferior.

El Hospital Comarcal Francisco de Borja de Gandía dispone de 250 camas con las que se da servicio al área sanitaria 11 de la Comunidad Valenciana, donde residen unos 250.000 habitantes. El servicio de Medicina Interna se compone de 20 facultativos incluyendo las especialidades de Neurología, Neumología, Enfermedades digestivas, Cardiología y Endocrinología.

A partir de los informes de alta generados en todo el hospital en la segunda quincena de enero del 2002, se recogieron los siguientes datos: Servicio, médico prescriptor, número de fármacos, proporción de ellos en los que existe alternativa como EFG disponible y porcentaje prescrito como tal. Se incluyeron todas las altas generadas en Medicina Interna, hospitalización a domicilio, Traumatología, Cirugía General, Urología, Otorrinolaringología, Ginecología y Oftalmología. En Puertas de Urgencias se analizaron los 25 primeros informes de alta de la mañana.

La comisión de Farmacia y Terapéutica elaboró una nota de información sobre el entorno legal y las EFG disponibles, que se difundió por correo interno a todos los facultativos del hospital en la segunda quincena de febrero.

En el Servicio de Medicina Interna se analizó también el perfil presciptor de cada facultativo, redactándose la hoja informativa personalizada, donde se destacaban los medicamentos habitualmente pautados que tenían su equivalente genérico. La distribución se realizó en las mismas fechas, pero de forma personal, con una breve explicación verbal del contenido y las motivaciones en la elaboración de dicha nota informativa, al tiempo que se preguntaba sobre su opinión sobre la prescripción de EFG.

En la segunda quincena de abril del 2002 se repitió la recogida de datos como la descrita. En el Servicio de Medicina Interna se realizó además otra recogida de datos en la primera quincena de marzo, es decir, inmediatamente después de la campaña de promoción.

\section{RESULTADOS}

Se revisaron un total de 1.545 informes de alta, 371 procedentes de Medicina Interna y 700 generados en puertas de urgencias. Se analizaron 3.460 fármacos prescritos.

El $42 \%$ de la medicación prescrita podría haberse pautado como EFG por tener su equivalente $(22 \%$ en el Servicio de Medicina Interna). El 93\% de estos posibles genéricos ser recomendaron desde 5 Servicios: Urgencias, Medicina Interna, Hospitalización a Domicilio, Traumatología y Cirugía.

Las posibilidades y el porcentaje de utilización en estos servicios en la primera toma de datos se muestran en la tabla I. Es destacable las amplias posibilidades y sin embargo la escasa utilización en Servicios como Cirugía y Traumatología, por la elevada representación de analgésicos o antinflamatorios de los que existe el equivalente como EFG.

\section{TABLA I}

DISTRIBUCIÓN POR SERVICIOS DEL NÚMERO Y PORCENTAJES DE POSIBLES EFG, Y LA PROPORCIÓN PAUTADA COMO TAL ANTES DE LA INTERVENCIÓN

\begin{tabular}{lccc}
\hline & Posibles genéricos & $\begin{array}{c}\text { \% respecto al total } \\
\text { de prescripciones }\end{array}$ & $\begin{array}{c}\% \text { prescrito } \\
\text { como EFG }\end{array}$ \\
\hline Medicina Interna & 125 & 20,5 & 21,6 \\
UHD & 77 & 41,4 & 16,3 \\
Urgencias & 83 & 22,7 & 23,1 \\
Cirugía & 40 & 68,3 & 2,4 \\
Traumatología & 48 & 47,1 & 0 \\
\hline
\end{tabular}

Tras la nota informativa no se modificaron de forma significativa en ninguno de los servicios los porcentajes de utilización EFG sobre el total de los posibles (Tabla II).

\section{TABLA I}

\begin{tabular}{|c|c|c|c|}
\hline \multicolumn{4}{|c|}{ TABLA II } \\
\hline \multicolumn{4}{|c|}{$\begin{array}{l}\text { DISTRIBUCIÓN POR SERVICIOS DEL NÚMERO Y } \\
\text { PORCENTAJES DE POSIBLES EFG, Y LA PROPORCIÓN } \\
\text { PAUTADA COMO TAL AL MES DE LA INTERVENCIÓN }\end{array}$} \\
\hline & Posibles genéricos & $\begin{array}{l}\text { \% respecto al total } \\
\text { de prescripciones }\end{array}$ & $\begin{array}{l}\% \text { prescrito } \\
\text { como EFG }\end{array}$ \\
\hline Medicina Interna & 92 & 22 & 27,8 \\
\hline UHD & 33 & 69 & 21,4 \\
\hline Urgencias & 155 & 32 & 5,8 \\
\hline Cirugía & 49 & 69 & 11,4 \\
\hline Traumatología & 44 & 44 & 0 \\
\hline
\end{tabular}

En Medicina Interna, donde la intervención se realizó de manera personalizada si se obtuvo un significativo incremento inmediato, del $21 \%$ se paso al $156,8 \%$ (RR 0,38 IC al $95 \%$ $0,26-0,55)$ pero que tendía a desvanecerse al mes $(27 \%)$ perdiendo la significación estadística (Fig. 1).

Las principales razones para la no-prescripción de EFG referidas por los 20 facultativos del Servicio de Medicina Interna fueron la falta de motivación $(40 \%)$, el no recordar o conocer las EFG (40\%) y /o la desconfianza en estos productos $30 \%$.

\section{DISCUSIÓN}

La regulación de las especialidades farmacéuticas genéricas se ha articulado en los últimos 5 años con distintas normativas de ámbito nacional o autonómico, con la incorporación progresiva de principios activos (1-3).

Aunque se ha descrito un aumento progresivo en la utilización de las EFG, su implantación es todavía relativamente 


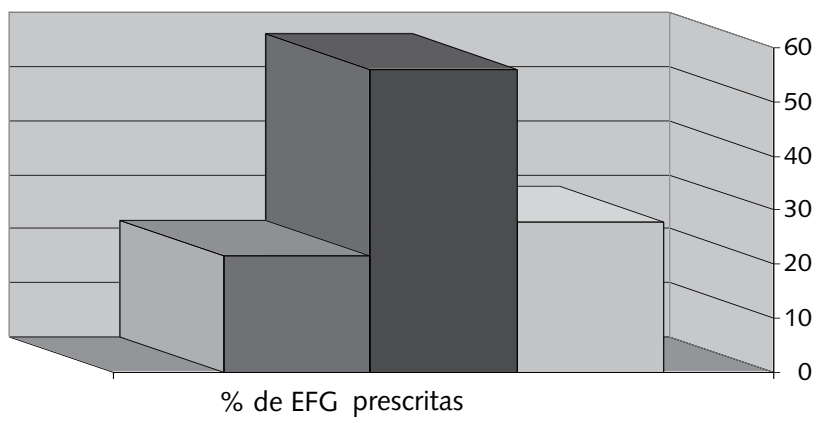

$$
\begin{aligned}
& \square \text { Basal } \\
& \square \text { Semana } \\
& \square \text { Mes }
\end{aligned}
$$

Fig. 1. Porcentaje de prescripción como EFG sobre el posible teórico de los que existe equivalente genérico previo a la intervención, a la semana y al mes, en el Servicio de Medicina Interna.

escasa (4-7) probablemente por razones complejas que incluyen la escasa tradición en nuestro medio, la falta de información de médicos y pacientes que genera desconfianza (8), la competencia desigual con las compañías farmacéuticas, etc.

En Atención Primaria se han realizado esfuerzos de infor- mación al usuario (8) y al médico prescriptor (9), incluso con incentivaciones concretas (10) que se han mostrado efectivas, sobre todo sí se plantean como estrategias mantenidas en el tiempo.

Las experiencias comunicadas en el entorno de atención hospitalaria son más limitadas y los porcentajes de utilización bajos $(11,12)$. Las posibilidades de intervención podrían ser sin embargo muy relevantes por la importancia del peso directo y de las prescripciones delegadas en atención primaria, así como por el incremento progresivo de las EFG disponibles. Las modificaciones posteriores de las pautas hospitalarias generan siempre cierto malestar en el paciente así como el desgaste del médico de Atención primaria, que se podrían evitar mediante el incremento en el porcentaje de utilización de EFG en los informes de alta.

En nuestra experiencia el resultado de campañas de promoción generales fue escaso. Pero incluso una información personalizada tan sólo obtuvo una eficacia relevante inmediata que se desvaneció progresivamente.

Además de mejorar la información respecto a la disponibilidad y eficacia de las EFG, las intervenciones dirigidas a potenciar su uso deberían también contemplar la implicación del prescriptor en el control del gasto mediante políticas de incentivación que deben articularse, tanto en la atención primaria como en la especializada.

\section{Bibliografía}

1. Ley $13 / 1996$ de 30 Diciembre en Medidas Fiscales, Administrativas y de Orden Social. Modificaciones de la Ley 25 /1990 de 20 Diciembre, del Medicamento. BOE $\mathrm{n}^{\mathrm{a}}$ 315, de 31 de Diciembre de 1996.

2. Real Decreto 1035/1999 de 18 Junio por el que se regula el sistema de precios de referencia en la financiación de medicamentos con cargo a fondos de la Seguridad Socia o a fondos estatales afectos a la sanidad. BOE $n^{a} 154$ de 29 Junio de 1999.

3. Carné X, Díez M, García-Alonso F. Las Especialidades farmaceúticas genéricas en España. Med Clin (Barc) 1999; 112 (9): 337-339.

4. Díaz A, López A. Prescripción de genéricos en el ärea de Salud de Zamora. Beneficios para el usuario y el Sistema Nacional de Salud. Aten Primaria 1997; 20: 499-504.

5. Verdejo A, López-Lázaro L, Rodríguez C, Piñeriro B, Pereira ML. Introducción de las especialidades farmaceúticas genéricas en Galicia. Aten Primaria 1999; 528-532.

6. Torralba M, Gilabert A, Peláez J, Faixedas MT. Implantación de medicamentos genéricos (EFG) en España y Cataluña: posibilidades de aho-

rro. Aten Primaria 2000; 26: 38-51.

7. Siles M, Goldaracena M. Medicamentos genéricos en España en España: primera etapa.Atención Primaria 2000; 26: 162-168.

8. Casado S, Sagardui JK, Lacalle M. Sustitución de medicamentos de marca por genéricos en la consulta de atención primaria. Atención Primaria 2002; 30: 343-349.

9. Calvo MJ, Iñesta A. Impacto de una estrategia de intervención en la prescripción de genérticos en un área de atención primaria. Aten Primaria 1999; 23: 419-424.

10. López-Picazo JJ, Sanz JA, Bernal JM, Sánchez JF. Evaluación, mejora y monitorización de la prescripción de medicamentos genéricos. Aten Primaria 2002; 29: 397-406.

11. Muñoz L, Ortego N, Canora J, Parra J, Gomez FJ, De la Higuera J. Prescripción de especialidades farmaceúticas genéricas en informes de alta de un hospital universitario. Med Clin (Barc) 2001; 116: 416-417.

12. Sicras A, Navarro R. Prescripción ambulatoria de medicamentos genéricos en un hospital Comarcal. Med Clin (Barc) 2001; 117: 757. 